\title{
Users' Preferences for Telemedical Consultations
}

\section{Comparing users' attitude towards different media in technology-mediated doctor-patient- communication}

\author{
Shirley Beul ${ }^{12}$, Martina Ziefle ${ }^{1} \&$ Eva-Maria Jakobs ${ }^{2}$ \\ ${ }^{1}$ eHealth Group/ Communication Science, Human Technology Centre \\ ${ }^{2}$ Textlinguistics and Technical Communication, Institute of Linguistics and Communication Studies \\ RWTH Aachen University \\ Aachen, Germany \\ \{beul, ziefle\}@humtec.rwth-aachen.de, \{s.beul, e.m.jakobs\}@tk.rwth-aachen.de
}

\begin{abstract}
Within industrialized countries healthcare systems currently change to cope with the upcoming consequences of the demographic change. One of the most serious challenges is the maintenance of the area-wide supply chain of medical care despite the threatening shortage of physicians. In this context, telemedical services for communication between doctors and patients gain in importance. Crucial for the success of such electronic services is the choice of the medium, which must be appropriate for this special purpose of use and, finally, accepted by its users. In this paper, an exploratory survey was conducted to detect acceptance motives of five different media (face-to-face, telephone, videophone, video conference, interactive wall) in two different usage situations. 103 respondents participated (17-83 years). Findings show that for the standard case, a face-to-face consultation is still highly preferred compared to any telemedical applications. For emergency situations, participants' attitudes change: A telephone consultation was similarly well accepted than face-to-face communication. As the most comfortable service a face-to-face-consultation was corroborated, followed by the videophone consultation.
\end{abstract}

Keywords: ehealth, telemedical consultation, teleconsultation, consultation medium, doctor-patient-communication, user acceptance, user preference, interactive wall

\section{INTRODUCTION}

Meanwhile, the demographic change is a much-discussed phenomenon: Due to the postwar baby booms demographic disequilibria were created. The postwar generation consisted of an extremely large cohort of children compared to previous generations [1]. Taking the demographic imbalance, longevity among the elderly as well as improvements in healthcare into account, an increasing number of old and frail people has to be provided with healthcare in the near future [1] [2]. Therefore, aging societies have to manage problems as rising healthcare expenditures [3], or a higher demand for medical personnel [4] [5].

In order to cope with these upcoming consequences, healthcare systems of industrialized countries currently change. One of the most serious challenges is the maintenance of areawide supply of medical care despite the threatening shortage of physicians. For this, the integration of information and communication technology into the medical system has been identified as a promising solution [6]. In this context,

Funded by the Excellence Initiative of German federal and states governments telemedical services for the purpose of doctor-patientcommunication gain in importance. Firstly, they can facilitate an optimal allocation of the human resources of doctors [7], secondly, bridge the geographical separation between physician and patient without causing travelling costs and a waste of time for the patient (travelling time, waiting) [8], and thirdly, give patients the option of having doctoral advice remotely, independent of time and location [9].

Crucial for the success of such a service is the choice of the medium through which a medical consultation occurs [10]. As a consequence, the used information and communication technologies (ICT) must be appropriate for this special purpose of use and, finally, accepted by its users [11], which, consequently, is reflected in the health outcome (e.g. coping).

For conceptualizing a teleconsultation service, that is accepted and actually used by the target group, it is necessary to understand its prospective user group. Especially patients' requirements on and preferences for media must be detected and carefully integrated in the electronic designs. Furthermore, it is essential if patients' preferences depend on demographic user characteristics, like age, gender, current health status, or technology affinity. Apart from these factors, the impact of the usage situation is also relevant, in particular differences between standard case and emergency case.

Many projects dealing with electronic health services currently investigate potential and pitfalls of telemedical consultations and the nature of the medicated doctor-patient communication which is altered by the use if electronic media. They deal with effects of written and oral communication; likewise real time and time-shifted communication is considered. Unfortunately, most of the recent studies regard a consultation medium exclusively in isolation [12] [13] [14], instead of comparing different media and devices respectively. Hence, it is unclear, which medium users prefer for an ICTbased medical encounter, if they would have the choice. Also, it is not understood so far to which extent the choice for an electronic medium mediating the doctor-patient communication is influenced by the situation, in which patients communicate with doctors.

To bridge this informational gap, acceptance motives of four different devices for real time communication (telephone, videophone, video conference, interactive wall) are examined in this research. The medium face-to-face was also considered, 
as the conventional and well-known medium (baseline). It was integrated as an item in the media comparison of the study in order to investigate how important the traditional form of medical encounter still is in the era of electronic health services. The users' preferences were investigated concerning their age, gender, health status, and technology affinity. Moreover, preferences for two different usage situations (standard case, emergency case) were detected.

\section{Methodology}

\section{A. Participants}

103 participants, aged between 17 and 83 years, took part in this study $(\mathrm{M}=49.97, \mathrm{SD}=17.93) .50 .5 \%$ of the sample are female, $48.5 \%$ male, $1 \%$ is unknown. To investigate if preferences for certain media differ depending on age and the affiliation to a certain technology generation, the sample was split in three age groups referring to three different technology generations: The first group is aged between 17 and 35 years $(\mathrm{M}=28.85, \mathrm{SD}=4.89,51.5 \%$ female $/ 48.5 \%$ male $)$, the second between 36 and 60 years $(\mathrm{M}=51.27, \mathrm{SD}=6.99,60.0 \%$ female/ $40.0 \%$ male), and the third between 61 and 83 years $(\mathrm{M}=67.76, \mathrm{SD}=5.95,43.2 \%$ female/ $56.8 \%$ male $)$.

Besides age, the respondents' state of health was paid attention. Mainly older people $(50+)$ were addressed because the prevalence for chronic diseases increases with rising age [15]. Potential participants were recruited in the authors' social networks as well as in pertinent online forums, self-help groups for chronically ill patients (prevailing cardiacs), and senior online communities. Finally, $60.2 \%$ of the respondents $(n=62)$ stated to be healthy. This group is aged between $17-83$ years, $(\mathrm{M}=45.45, \mathrm{SD}=17.57,49.2 \%$ female/ $50.8 \%$ male $) . \mathrm{N}=37$ (35.9\%) affirmed being chronically ill, which requires seeing one or more doctors regularly. The age range in the chronically ill group is $21-82$ years $(\mathrm{M}=57.97, \mathrm{SD}=17.57)$. Among the chronically ill persons, $48.6 \%$ are female, $51.4 \%$ male.

Owing to the fact that different ICT are assessed in this study, respondents' attitude towards technology must be considered as well. Based on the variable technology affinity, the sample was split in two groups: $43.8 \%$ of the respondents were assigned in the group with a low technology affinity, $56.3 \%$ belong to the high technology affinity group. Concerning the three age groups, the relation between low and high technology affinity was almost in balance in all groups. Only the youngest group showed a predominantly high technology affinity $(71.9 \%)$. In contrast to this, the gender distribution is very unequal: $81.0 \%$ of the group with low technology affinity are female, only $19.0 \%$ male (group with high technology affinity: $24.5 \%$ female, $75.5 \%$ male). Between the self-assessment of healthy and chronically ill people concerning technology affinity no significant differences were found (high technology affinity: 64.8\% healthy, 35.2\% chronically ill; low technology affinity: $59.5 \%$ healthy, 40.5 chronically ill).

\section{B. The Questionnaire}

In order to get a deeper insight in users' medial preferences for telemedical consultations, a method had to be chosen which allows collecting comprehensive opinions of prospective users, as well as a large number of respondents. For this reason, the questionnaire method was applied.

The questionnaire was organized in three different sections: The first part consisted of a query of demographic and health data with respect to participants' age, gender, and health status. Section two was about participants' general attitude towards technology. Instead of measuring user experience (frequency of use, perceived ease of use) participants' technology affinity was explored. This variable was modeled out of test persons' self-assessment in five categories: technology enthusiasm, interest in technology, distrust of technology, ability to use technology, and technical understanding. In the third section four telemedical services for remote doctor-patientcommunication were delineated. Each of them focused another consultation medium: a telephone, a video conference on an computer monitor, a videophone, and a new ICT, a so-called interactive wall, which refers to a wall-sized multi-touch display and which was lately introduced in telemedical scenarios [16] [17]. All devices were explained with respect to the presentation of the interlocutor, in particular, if, how and which part of the interlocutor is visualized depending on the device. Furthermore, it was specially emphasized that the presentation was reciprocal: One interlocutor is similarly presented for the other, as the other receives him/her verbally, and, depending on the device, also visually. In order to illustrate the functionality of the devices, each of them was depicted (Fig. 1).

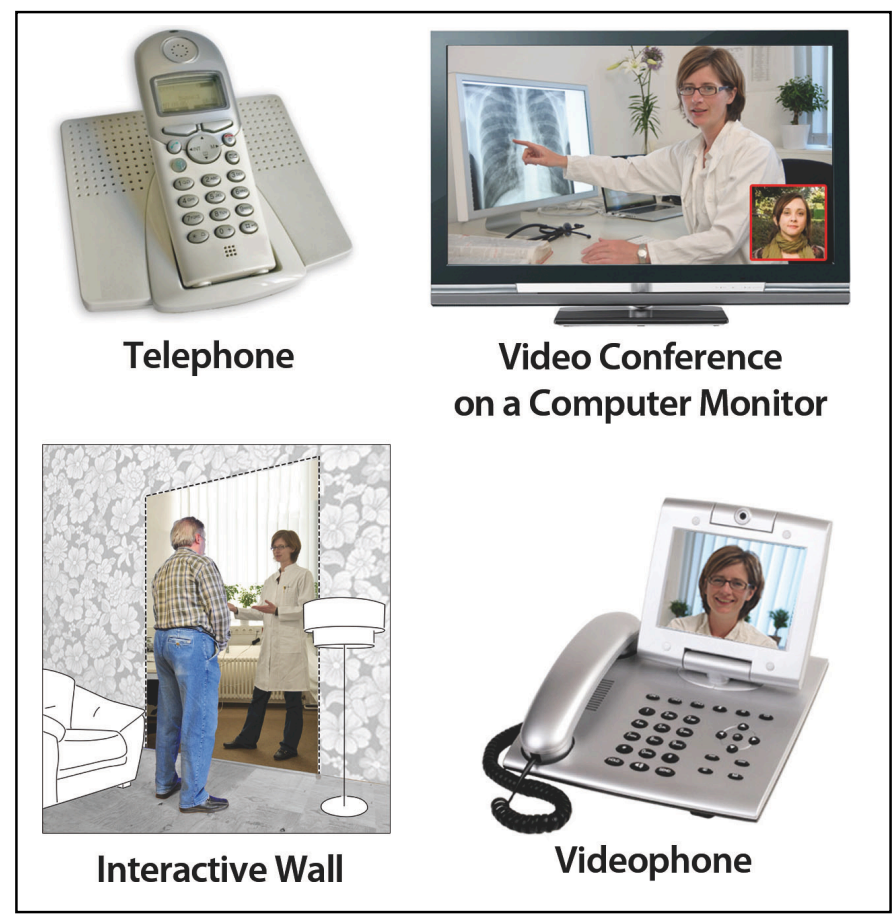

Figure 1. Visualization of consultation media in the questionnaire

Afterwards, a query about five media was added. Respondents answered questions by favoring one of the four telemedical services, respectively devices, or the medium through which a medical encounter usually occurs: a face-toface talk. It was integrated as an item in the media comparison of the study to investigate how important this traditional medical encounter still is in the era of eHealth. The query 
implied questions to measure the general willingness to use media for a (tele)medical encounter depending on the usage situation. Respondents' preferences for a (tele)medical encounter in the standard and in a emergency case were measured. An emergency encounter was defined as a situation, in which the respondent needs advice of her/his doctor urgently. It was also emphasized that in both situations the consultation would be executed by a physician respondents already know, e.g. 'their' (family) doctor. Moreover, it was assessed which medium facilitates the most comfortable (tele)medical encounter.

\section{Research Variables}

In this study, respondents' age, gender, health status, and technology affinity were considered as independent variables. Three age groups were formed in order to detect age-specific preferences for media, and to compare younger, middle, and older aged people as well as three different technology generations (computer, household and early technical generation, [18] [19]. Gender is regarded for contrasting data of female and male participants, which could have different communication styles and attitude toward the use of electronic media in the doctor-patient communication [20] [21]; health status for investigating differences between healthy and chronically ill respondents [22]. Technology affinity was assessed in order to learn if users' general attitude towards technology influences medial preferences [23]. As dependent variables, the willingness to use certain media for a medical encounter a) in the standard case, and b) in case of emergency were measured. Additionally, the comfort of the media in the standard case was considered as a dependent variable.

\section{RESULTS}

\section{A. Medial preferences for a medical encounter in the standard case}

Comparing the five types of medical encounter directly, respondents have a clear favorite. According to participants' opinion, the face-to-face consultation is with $82.7 \%$ by far the most preferred type of medical encounter. Only $17.7 \%$ chose ICT based services $(6.3 \%$ videophone, $4.2 \%$ video conference, $4.2 \%$ interactive wall, $3.1 \%$ telephone) (Fig. 2).

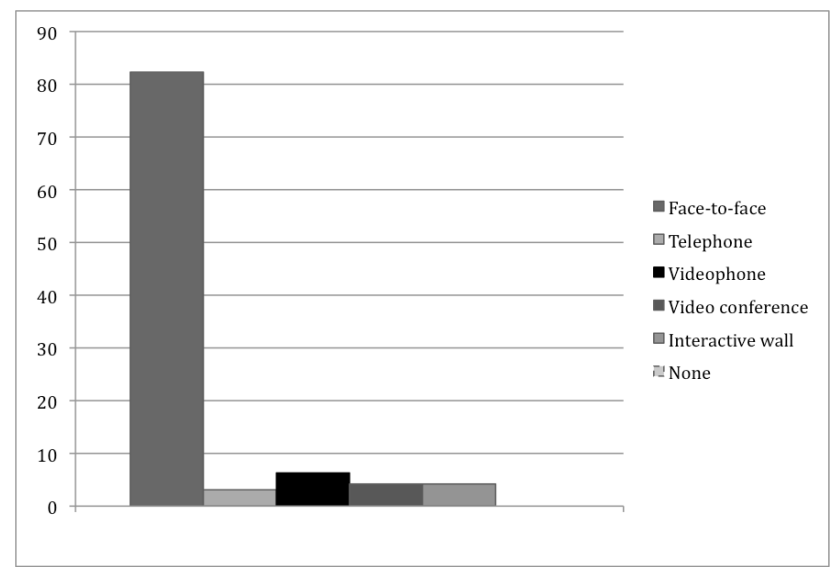

Figure 2. Medial preference for a medical encounter in the standard case (in \%)
If the comfort of these five types of medical encounter is assessed, face-to-face is also the most common preference $(42.1 \%)$. However, the overall distribution of the collected votes changed: $53.7 \%$ of the sample chose telemedical services as the most comfortable medium. $24.2 \%$ respondents opt for the videophone service, $11.6 \%$ for the interactive wall service, and $7.4 \%$ for the video conference service. $4.2 \%$ voted for none of the options (Fig. 3).

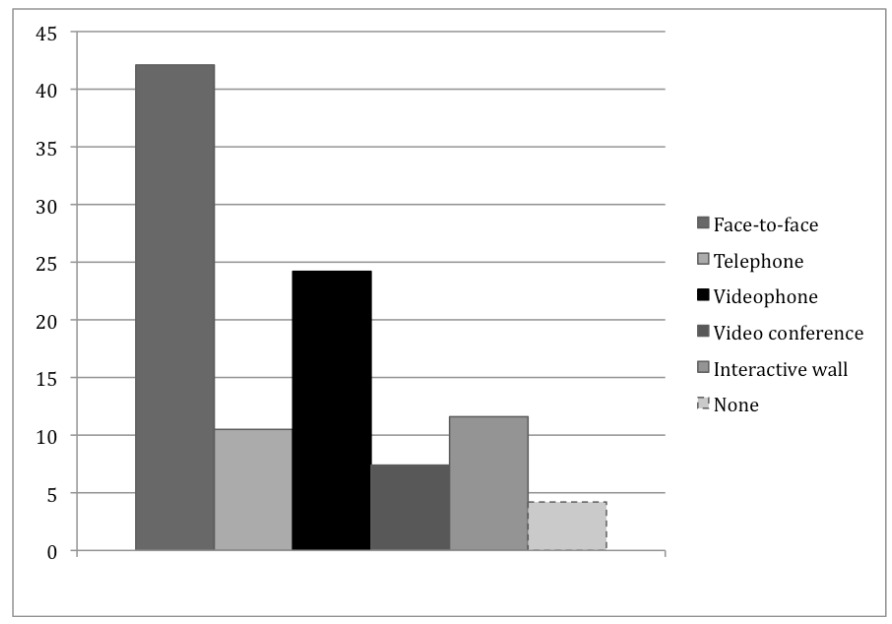

Figure 3. Medial preference considering the comfort aspect (in \%)

Among the three age groups, preferences considering the comfort aspect differ completely. The oldest group favored the videophone service $(38.9 \%)$. It is closely followed by the faceto-face encounter, which was chosen by one third of the older respondents. $13.9 \%$ opt for the video conference service, $5.6 \%$ for the telephone as well as the interactive wall. $2.8 \%$ picked 'None' (Fig. 4).

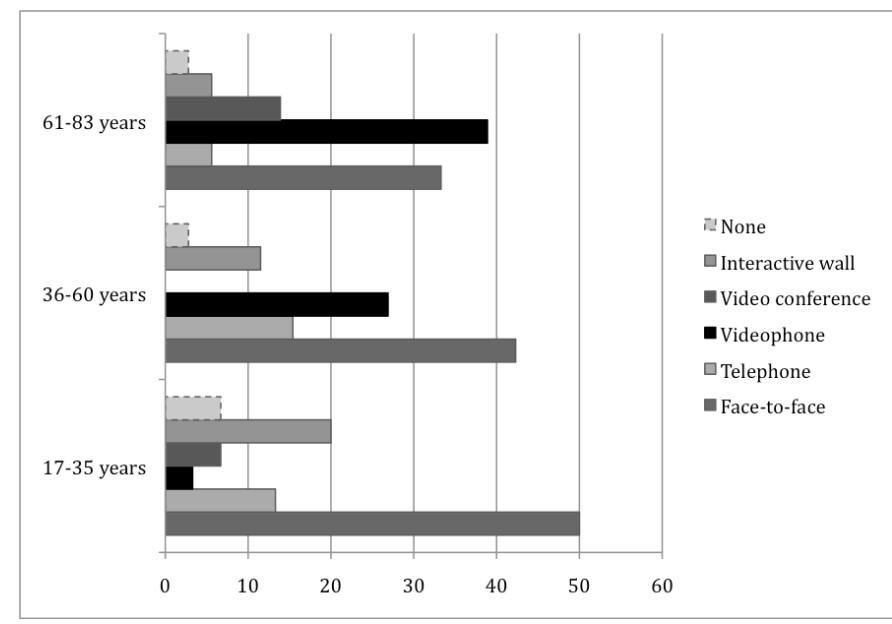

Figure 4. Medial preference of three age groups considering the comfort aspect (in \%)

The middle-aged group prefers to talk to a doctor face-toface $(42.3 \%) .26 .9 \%$ of them voted for the videophone encounter, $15.4 \%$ for the telephone, $11.5 \%$ for the interactive wall, and $3.8 \%$ for none option. Interestingly, no representative of this group opt for the video conference service.

Half of the youngest group of this sample chose the face-toface-consultation. $20 \%$ of this group assessed the interactive 
wall consultation as the most comfortable type of medical encounter.13.3\% opt for the telephone, $6.7 \%$ the video conference, and $3.3 \%$ the videophone. 'None' was chosen by $6.7 \%$ (Fig. 4).

Regarding the comfort aspect under consideration of gender, no major differences were found: Both genders believe the face-to-face talk with a physician is the most comfortable type of medical consultation (34.8\% female, $47.9 \%$ male), followed by the videophone consultation $(30.4 \%$ female, $18.8 \%$ male). $15.2 \%$ of female participants prefer the telephone, while only $6.3 \%$ of male respondents would use it. $13.0 \%$ females and $10.4 \%$ males opt for the interactive wall encounter. The video conference was chosen by $10.4 \%$ of the men, and $4.3 \%$ of the women. $2.2 \%$ females, and males picked 'None' (Fig. 5).

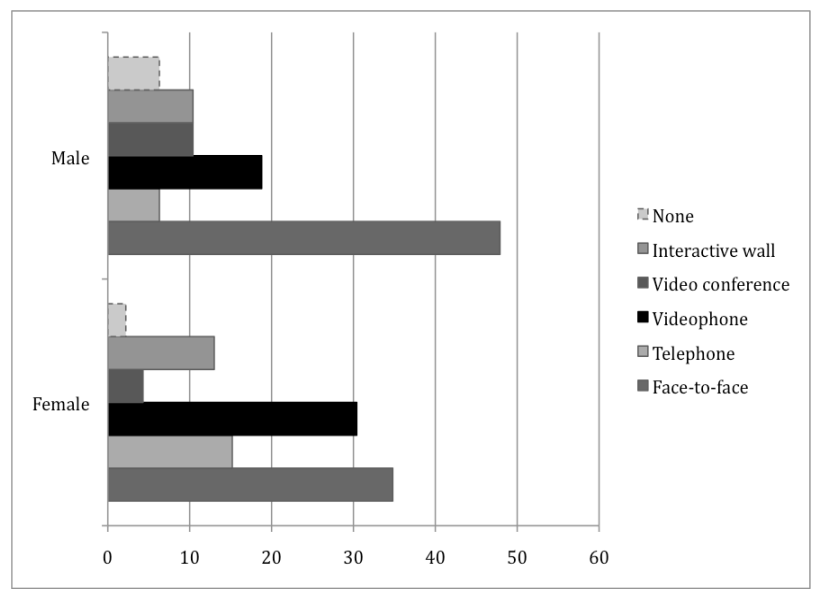

Figure 5. Gender aspects: medial preferences considering comfort (in \%)

According to the choice of the chronically ill, face-to-face is the most comfortable alternative $(41.7 \%)$, but closely followed by the videophone with $38.9 \%$. $8.3 \%$ of the chronically ill respondents opt for the video conference talk, $5.6 \%$ for the interactive wall encounter, and $2.8 \%$ for the telephone consultation. $2.8 \%$ chose 'None'. Figure 6 shows the outcomes.

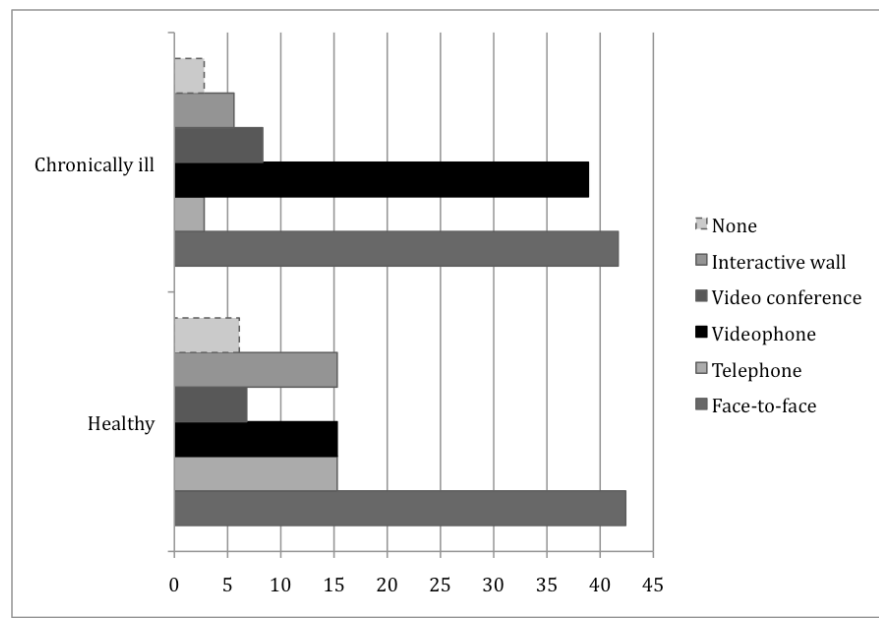

Figure 6. Health status of participants: medial preferences considering comfort (in \%)
The healthy also favored the face-to-face encounter (42.4\%), however, $52.7 \%$ preferred ICT based solutions (telephone, videophone, interactive wall: each $15.3 \% ; 6.8 \%$ video conference). 5.1\% went to 'None' (Fig. 6).

Medial preferences differ when investigating participants' technology affinity: People with a low technology affinity assessed the videophone service as the most comfortable $(40.0 \%)$, while $32.5 \%$ voted for the face-to-face talk. The telephone encounter received $15 \%$ of the votes of respondents with low technology affinity.

The video conference and the interactive wall obtained each $5.0 \%$. 2.5\% picked 'None'. Consequently, 65\% of the group with a low technology affinity opts for ICT based solutions. Among the participants with a high technology affinity, a clear preference for the face-to-face consultation is obvious (48.1\%). Telemedical services were chosen only by $46,7 \%(16.7 \%$ interactive wall, $13.0 \%$ videophone, $9.3 \%$ video conference, $7.4 \%$ telephone). 'None' was chosen by $5.6 \%$ (Fig. 7).

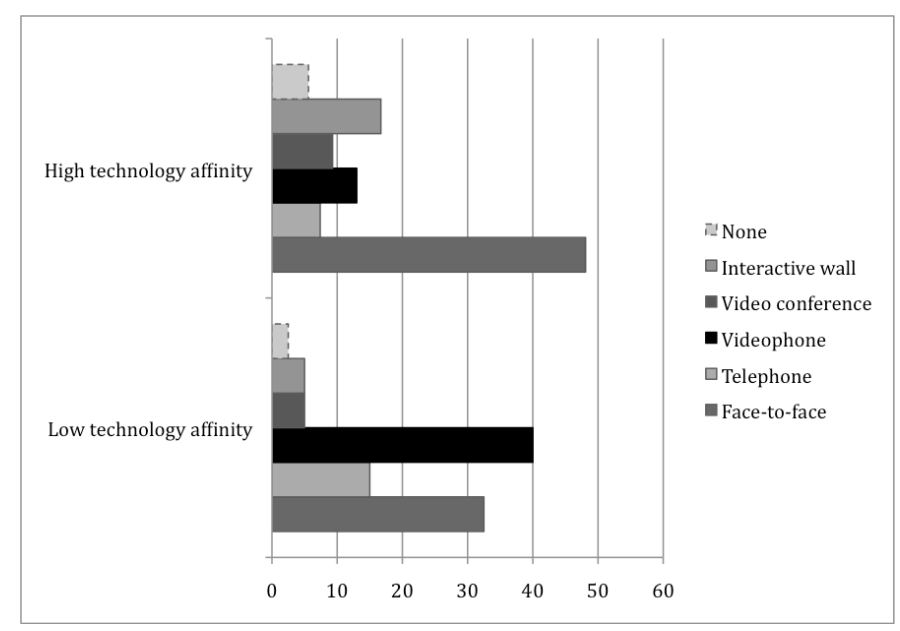

Figure 7. Technology affinity of participants: medial preferences considering comfort (in \%)

\section{B. Medial preferences for a medical encounter in case of emergency}

Apparently, the usage situation has an impact on participants' preferences for the type of medical encounter. Whereas the face-to-face-consultation $(82.7 \%)$ was favored in the standard case, only $30.5 \%$ of the respondents opt for it in case of emergency. Thus, telemedical services are preferred in case of emergency $(67.4 \%)$. The most popular service is the telephone consultation in this scenario $(33.7 \%)$, followed by the videophone $(14.7 \%)$, the video conferencing $(11.6 \%)$, and the interactive wall (7.4\%). 2.1\% picked 'None' (Fig. 8).

In particular, the oldest groups first choice in case of emergency is the telephone consultation (30.6\%), second choice is the face-to-face talk $(25.0 \%) .22 .2 \%$ opt for the videophone, $19.4 \%$ for the video conference, and $2.8 \%$ for the interactive wall. $2.8 \%$ voted for 'None'.

Among the middle-aged group, the medium telephone is also preferred (34.6\%), followed by the face-to-face consultation $(23.1 \%)$. The rest of the votes of this group are distributed across the remaining media as follows: $19.2 \%$ indicate to select videophone, $15.4 \%$ opt for interactive wall, 
$3.8 \%$ of the sample selects video conferencing. 'None' was picked by $3.8 \%$.

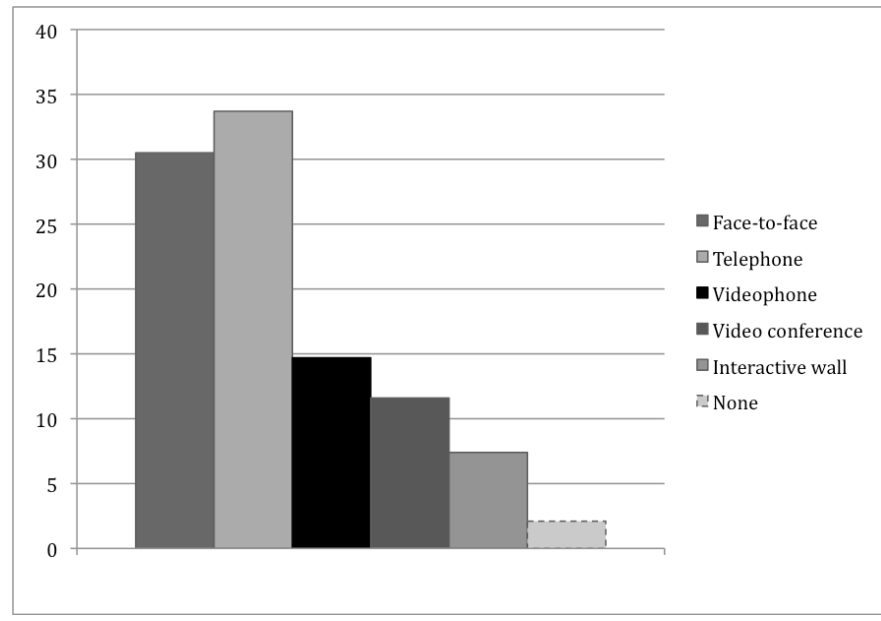

Figure 8. Medial preference for a medical encounter in case of emergency (in \%)

However, the face-to-face encounter is even preferred by the youngest group in case of emergency $(43.3 \%)$. The telephone service $(40.0 \%)$, the video consultation $(6.7 \%)$, and the interactive wall $(6.7 \%)$ follow in the ranking. $3.3 \%$ went to 'None'. No representative of this age group favored the videophone (Fig. 9).

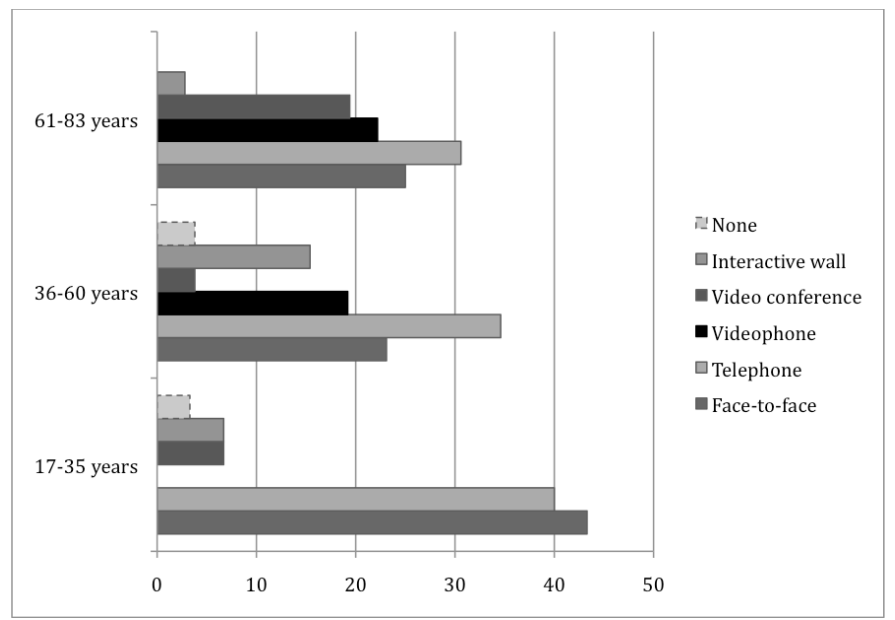

Figure 9. Medial preference of three age groups in case of emergency (in \%)

Also in this usage situation, gender differences barely exist: Both genders first choice is the telephone consultation (female $32.6 \%$, male $35.4 \%$ ), their common second choice is the faceto-face encounter (female $28.3 \%$, male $31.3 \%$ ). More women $(23.9 \%)$ than men $(6.3 \%)$ favor the videophone service. Regarding the video conference service, it is vice versa: More males $(14.6 \%)$ opt for it than females $(8.7 \%)$ did. $4.3 \%$ women, and $10.4 \%$ men chose the interactive wall. $2.2 \%$ (female), respectively $2.1 \%$ (male) went to 'None' (Fig. 10).

In case of emergency, the chronically ill prefer as well as the healthy the telephone consultation (chronically ill 30.6\%, healthy $35.6 \%$ ). The face-to-face encounter is picked by $27.8 \%$ of the chronically ill, and by $32.2 \%$ of the healthy respondents.

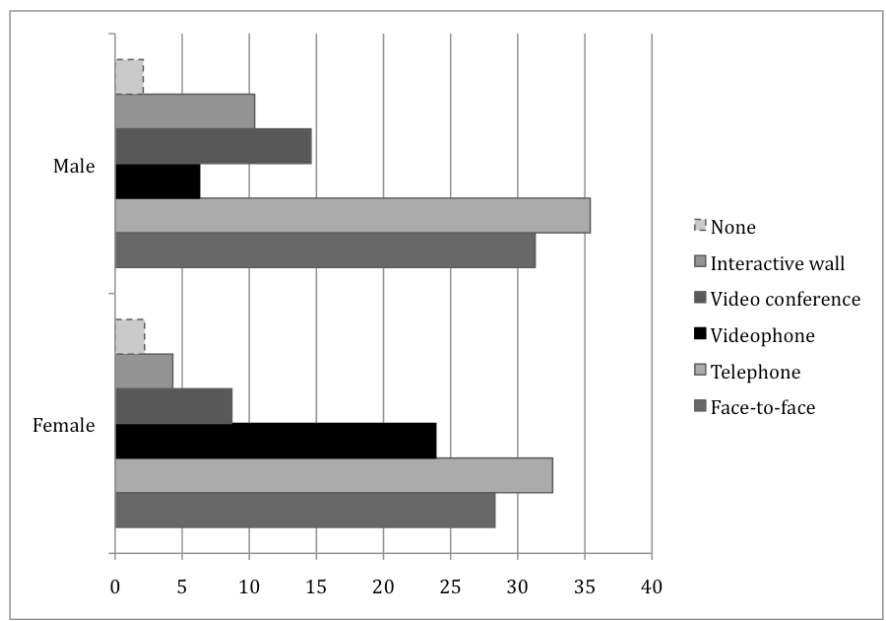

Figure 10. Gender aspects: medial preferences in case of emergency (in \%)

Among the chronically ill, the videophone is chosen by $27.8 \%$, but only by $6.8 \%$ of the healthy. The video conferencing as communication medium achieved almost the same amount of votes: $11.9 \%$ of the healthy, $11.1 \%$ of the chronically ill chose it. $10.2 \%$ of healthy people favor the interactive wall in case of emergency, while only $2.8 \%$ of chronically ill pick this alternative (Fig. 11).

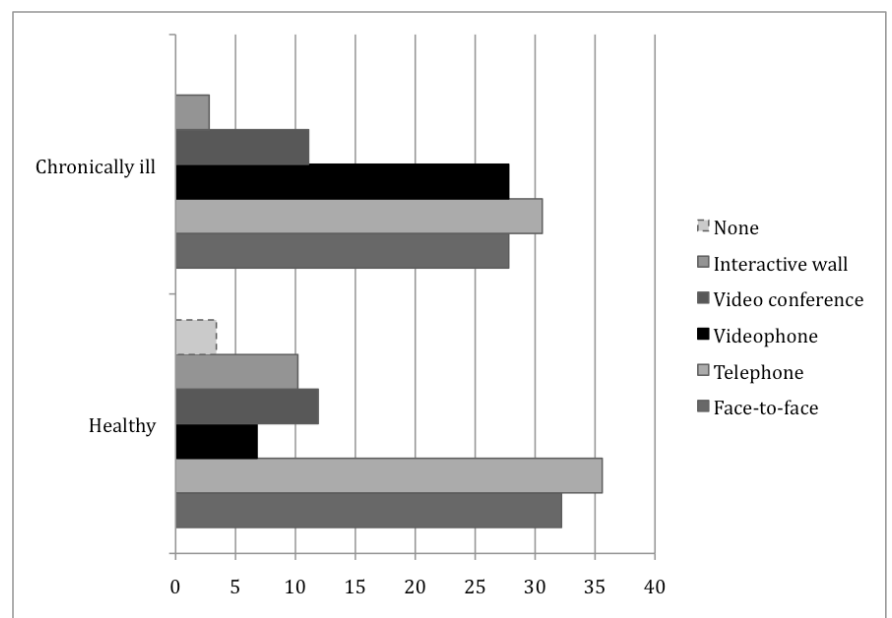

Figure 11. Health status of participants: medial preferences in case of emergency (in \%)

By comparing participants' medial preferences in case of emergency under consideration of their technology affinity, significant differences were found: People with a high technology affinity favor receiving medical support by their doctor through the telephone in this scenario $(32.5 \%)$, closely followed by the face-to-face consultation. The other ICT based services were picked less $(13.0 \%$ for the video conference, $11.1 \%$ for the interactive wall $5.6 \%$ for the videophone).

The group with the low technical affinity preferred the telephone encounter as well (32.5\%), and also closely followed by the face-to-face talk $(27.5 \%)$. Furthermore, the videophone achieved the same amount of votes $(27.5 \%)$. The video conferencing service was favored by $10.0 \%$ of this group. Only $2.5 \%$ chose the interactive wall. In Fig. 12, the descriptive outcomes are visualized. 


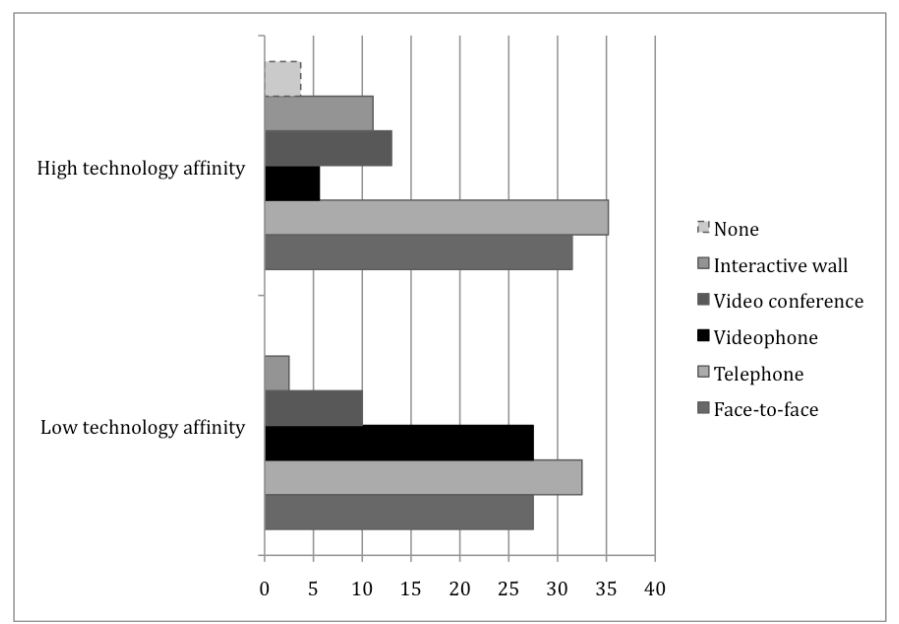

Figure 12. Technology affinity of participants: medial preferences in case of emergency (in \%)

\section{DISCUSSION AND CONCLUSION}

Findings on the medial preferences show that a face-to-face consultation is still highly preferred compared to telemedical applications in the standard case. Regardless whether respondents were older or younger, female or male, healthy or not, the traditional type of medical encounter is the first choice. Even while regarding respondents' technical affinity, no significant differences were found.

This fact is attributed to the impact of electronic health services in these times. In the majority of western countries, the need of telemedical services and their importance for a comprehensive and economic health care is uncontroversial. So far, however, and this is a critical point, electronic services have been poorly implemented in the existing healthcare systems so far [24] [25]. Most of these applications are only in the early stadium of prototyping and testing [26]. Consequently, there is a lack of user experience with telemedical services among patients, users respectively [27] 28]. So, one possible interpretation of this result is that people could only prefer what they have experienced so far, at least for the standard case.

In addition, some of them also complained about an obvious fact in the additional comments: A physical examination by a physician (e.g. auscultation) is not possible through telemedical services. Thus, these services can be appropriate for talk-based encounters (e.g. discussing vital data in monitoring checkups).

Considering the comfort aspect, the face-to-face consultation is also most widely chosen, but the electronic health services gain in attractiveness for the patients as well. At least, more than half of the respondents assessed a telemedical solution as the most comfortable type of medical encounter. This can support the previous hypothesis, too. The telemedical services facilitate bringing physician and patient together without wasting the patient's time (travelling, waiting) [29]. Besides, the patient can have doctoral advice remotely, independent of time and location. Obviously, these facts enhance patients' comfort. Here again, a lack of user experience could be the reason for this result [27] [28].
Another possible explanation could be that comfort means something different to users than the obvious advantages of the ICT based medical encounters. In the additional comments, some of them stated that visiting their doctor "is something personal" and that the delineated telemedical services were "too anonymous". This findings confirms outcomes of Stanberry [28] who concludes his paper on barriers and opportunities of telemedicine in the $21^{\text {st }}$ century as early as in the year 2000:

,The sceptics of telemedicine are right to insist that it should only be used where a clear case can be made for its implementation based upon clinical need, costbenefit or the improvement of the quality of a health service, because the inevitable result of our overenthusiastic development of technologies for distant consultation and treatment - no matter how fast we can be treated in the future - will be the loss of the one intangible thing that we most value about traditional health care: the comfort and compassion human beings can only truly bring each other when they are face to face" (Stanberry, 2000, p. 627).

From the patient's point of view, subsequently, comfort could be operationalized differently. Comfort could mean having their doctor in the immediate proximity, wherefore the patient could just go to his/her place and see her/him face-toface instead of her/his picture transmitted on a screen. It can also mean the physicians' (familiar) reliability, which is not affected by the reliability of any ICT. In order to model the factor comfort in the context of telemedicine properly, and hence, to understand patients' needs and requirements on telemedical service, it is necessary to explore patients system of values in additional (qualitative) research (e.g. interview studies).

For emergencies, participants' preferences toward the appropriate communication medium changes: A telephone consultation was similarly accepted as face-to-face communication. In total, telemedical services were preferred. This can be explained by the fact that time is the most crucial factor in case of an emergency. In this usage situation, patients want to be medically treated as soon as possible, and are more willing to receive healthcare support through telemedical applications than in the standard case. Actually, people with a low technology affinity begin to change their opinion and to think an ICT based consultation could be use- and helpful in case of emergency. Even when it is exclusively for the purpose of letting a doctor appear on the screen of a videophone just to calm the patient down, until an ambulance arrives only a few minutes later at her/his place.

In conclusion, telemedical services are a promising solution for maintaining the area-wide supply of healthcare at times when medical experts are rare, and an enormous amount of patients has to be treated medically. In this study, user characteristics influence acceptance motives to different degrees: Age-specific differences were more striking than gender, or health status effects (which is also linked to age). Technology affinity can play an important role as well, however, the usage situation determines patients' acceptance motive the most. 


\section{FUTURE WORK}

It is obvious that the exclusive focus on two general usage situations, the standard case and the emergency case, is insufficient for a comprehensive understanding of users' acceptance motives for the mentioned telemedical services. Crucial acceptance factors of these concepts - apart from user characteristics - have not been identified yet. For this reason, an additional questionnaire-study will be run, in which three different usage scenarios will be delineated to a more precise extend. To identify potential usage barriers, study respondents will assess usage conditions of each telemedical service.

\section{ACKNOWLEDGMENT}

We thank our participants, especially the older and ill ones, for spending their time on filling in our questionnaire so patiently. The Excellence Initiative of the German federal and state governments funded this research.

\section{REFERENCES}

[1] D.E. Bloom, and D. Canning, "Global Demographic Change: Dimensions and Economic Significance," Proceedings of the Symposium on Global Demographic Change: Economic Impacts and Policy Challenges, Federal Reserve Bank of Kansas City, August 2004, pp. 9-56.

[2] S. Leonhardt, M. Hexamer, and O. Simanski, "Smart Life Support: model-based design and control of life-supporting systems, “ J. Biomed Tech (Berl). 54 (5), October 2009, pp. 229-231.

[3] M. Seshamani, and A. Gray, "Time to death and expenditure: an improved model for the impact of demographic change on health care," J. Age and Ageing, 33 (6), 2004, pp. 556-561.

[4] K. Blum, and F. Löffert, "Ärztemangel im Krankenhaus - Ausmaß, Ursachen, Gegenmaßnahmen," Düsseldorf: Deutsches Krankenhaus Institut, 2009.

[5] S. Beul, S. Mennicken, M. Ziefle, and E.-M. Jakobs, "What happens after calling the ambulance: information, communication, and acceptance issues in a telemedical workflow," Proceedings of the International Conference on Information Society, June 2010.

[6] M. Ziefle, "Potential and pitfalls of age-sensitive technologies in the e-health field," Proceedings of the 1st European Conference on Ergonomics. Ergonomics in and for Europe. Quality of Life: Social, Economic and Ergonomic Challenges for Ageing People at Work, Brugge, October 2010.

[7] K. Yusof, K. H. B. Neoh, M. A. bin Hashim, and I. Inbrahim, "Role of Teleconsultation in Moving the Heakthcare System Forward," Asia Pac J Public Health 14(1), 2002, 29-34.

[8] E. A. Miller, "The technical and interpersonal aspects of telemedicine: effects on doctor-patient communication", J. Telemed Telecare, 9, 2003, pp. 1-7.

[9] R. L. Bashshur, "On the Definition and Evaluation of Telemedicine," J. Telemedicine, 1(1), 1995, pp. 19-30.

[10] E. A. Miller, "Telemedicine and doctor-patient-communication: a theoretical framework for evaluation," J. Telemed Telecare 8, 2002, pp. 311-318.

[11] S. Buck, "Nine human factors contributing to the user acceptance of telemedicine applications: a cognitive-emotional approach," J. Telemed Telecare 15, 2009, pp. 55-58.

[12] D. R. Parker, G. Demiris, and D. Porock, "The usability of videophones for seniors and hospice providers: a brief report of two studies," J. Comput Biol Med 35, 2005, pp. 782-790.

[13] C. LeRouge, M. J. Garfield, and A.R. Hevner, "Quality Attributes in Telemedicine Video Conferencing," Proceedings of the 35th Hawaii International Conference on System Sciences, January 2002.
[14] R. E. Izqueirdo, P. E. Knudson, S. Meyer, J. Kearns, R. Ploutz-Snyder, and R. S. Weinstock, "A Comparison of Diabetes Education Administered Through Telemedicine Versus in Person,“ J. Diabetes Care 26 (4), 2003, pp. 1002-1007.

[15] K. K. L. Ho, J. L. Pinsky, W. B. Kannel, and D. Levy, "The Epidemiology of Heart Failure: The Framingham Study," J. American College of Cardiology 22 (4), 1993, 6A-13A.

[16] M. Ziefle, C. Röcker, W. Wilkowska, K. Kasugai, L. Klack, C. Möllering, and S. Beul, "A Multi-Disciplinary Approach to Ambient Assisted Living". in E-Health, Assistive Technologies and Applications for Assisted Living: Challenges and Solutions, C. Röcker and M. Ziefle, Eds. Hershey, P.A.: IGI Global, pp. 76-93.

[17] M. Ziefle, C. Röcker, K. Kasugai, L. Klack, E.-M. Jakobs, T. SchmitzRode, P. Russell, and J. Borchers, "eHealth - Enhancing Mobility with Aging," in Roots for the Future of Ambient Intelligence, M. Tscheligi, B. de Ruyter, J. Soldatos, A. Meschtscherjakov, C. Buiza, W. Reitberger, N. Streitz, and T. Mirlacher, Eds. Adjunct Proceedings of the Third European Conference on Ambient Intelligence (AmI'09), pp. 25 28.

[18] M. Ziefle and W. Wilkowska, "Technology acceptability for medica assistance," in Proceedings of the 4th ICST/IEEE Conference on Pervasive Computing Technologies for Healthcare, 2010.

[19] W. Wilkowska and M. Ziefle, "User diversity as a challenge for the integration of medical technology into future home environments," in Human-Centered Design of eHealth Technologies: Concepts, Methods and Applications, M. Ziefle and C. Röcker, Eds. Hershey, PA, USA: IGI Global, 2011, pp. 95-126.

[20] O. Chorng-Shyong, and L. Jung-Yu, "Gender differences in perception and relationship among dominants of e-learning acceptance," Computers in Human Behavior 22 (5), 2006, pp. 816-829.

[21] V. Terzis, and A. A. Economides, "The acceptance and use of computer based assessment," Computers \& Education 56(4), 2011, pp.1032-1044.

[22] W. J. Winkelman, K. J. Leonard, P. G. Rossos, "Patient-Perceived Usefulness of Online Electronic Medical Records: Employing Grounded Theory in the Development of Information and Communication Technologies for Use by Patients Living with Chronic Illness,“ J. Am Med Inform Assoc 12 (3), 2005, pp. 306-314.

[23] M. Ziefle, A. K. Schaar, "Technical expertise and its influence on the acceptance of future medical technologies. What is influencing what to which extent?" in HCI in Work \& Learning, Life \& Leisure, USAB 2010, LNCS 6389, G. Leitner, M. Hitz, and A. Holzinger, Eds., Berlin: Springer, 2010, pp. 82-100.

[24] K. Walshe, and T.G. Rundall, "Evidence-based Management: From Theory to Practise in health Care," Milbank Quarterly 79 (3), 2001, pp. 429-457.

[25] L. C. Burtin, G. F. Anderson, and I.W. Kues, "Using Electronic Health records to help coordinate Care," Milbank Quarterly 82 (3), 2004, pp. 457-481.

[26] A. Obstfelder, K. H. Engeseth, and R. Wynn, "Characteristics of sucessfully implemented telemedical applications," Implementation Science 2 (25), 2007, 2:25 doi:10.1186/1748-5908-2-25.

[27] S. Beul, S. Mennicken, M. Ziefle, E.-M. Jakobs, D. Wielpütz, M. Skorning, and R. Rossaint, "The impact of usability in emergency telemedical services," in Advances in Human Factors and Ergonomics in Healthcare, V. Duffy, Ed., 2010, Boca Raton: CRC Press.

[28] R. Wears, and S. Perry, "Human factors and ergonomics in the emergency department," Ann Emergency Medicine 40 (2), 2002, pp. 206-212.

[29] M. Reddy, S. Paul, S., J. Abraham, M. McNeese, C. DeFlitch, and C. J. Yen, "Challenges to effective crisis management: Using information and communication technologies to coordinate emergency medical services and emergency department teams," Int J. Med Inform 78 (4), 2009, pp. 259-269.

[30] B. Stanberry, "Telemedicine: barriers and opportunities in the $21^{\text {st }}$ century,” J. Internal Medicine 247 (6), 2000, pp. 615-628. 